\title{
Are neutral Goldstone bosons initiating very energetic air showers and anomalous multiple-core structure as a component of cosmic rays?
}

\author{
Saul Barshay and Georg Kreyerhoff \\ III. Physikalisches Institut \\ RWTH Aachen \\ D-52056 Aachen \\ Germany
}

November 15, 2018

\begin{abstract}
We consider two recently accentuated, unusual empirical results concerning cosmic-ray events at high energies. We show that the possibility for a correlated explanation is provided by new dynamics which arises from collisions of a neutral Goldstone boson as a component of the highest-energy cosmic rays.
\end{abstract}

In this paper, we consider the hypothesis that there is a nearly-massless neutral Goldstone boson, $b^{0}$, which is a component of high-energy cosmic rays and which is initiating air showers [1, 2, 3] at the highest energies and possibly also anomalous multiple-core structure seen in photo-emulsion chamber experiments [4, 5, 6, 月, 8]. We point out and examine the consequences of new dynamics which arises from the assumption that there is an effective interaction originating in a neutral triangle anomaly [9, 10] involving $b^{0}$ and a neutral vector boson $Z^{\prime}$ (and/or a chirallyrelated neutral axial vector boson). This possibility may be related to the possibility that neutrino mass originates as a consequence of a spontaneously broken chiral symmetry at a low energy scale, $F<0.4 \mathrm{MeV}$. The strength of the effective interaction is $\left(\frac{g^{2}}{2 \pi^{2} F}\right)$ with $g^{2}$ assumed, for numerical illustration of the idea, to be of the order of 0.1 (i. e. like a strength for $Z$ ). $F^{F 1}$ The communication of $b^{0}$ with quarks is only via $Z^{\prime}$ exchange; among leptons, $Z^{\prime}$ likely couples only to neutrinos (like $b^{0}$ ). Then the mass of $Z^{\prime}$ may not be very much greater than that of $Z$. An actual value up to some hundreds of $\mathrm{GeV}$ does not affect our display of interesting dynamics, because we are considering cosmic-ray collisions at (c. m.) energies well above $m_{Z^{\prime}}$, i. e. $>10 \mathrm{TeV}$ for $b^{0}$-nucleon collisions $(>1 \mathrm{TeV}$ for an idealized $b^{0}$-quark collision).

Our main purpose is to clearly exhibit the relevance of the hypothesis to providing new dynamics bearing upon two long-standing puzzling aspects of cosmic-ray interactions at the highest energies. 1. The presence of a few "hadron-like" air shower events at primary energies estimated to be about $10^{20} \mathrm{eV}$. 11, 2] The data still is limited, but this is an energy above the Greisen-Zatsepin-Kuzmin cut-off 11, 12] for arrival of protons from sources at very great (cosmological) distances,

\footnotetext{
${ }^{F 1}$ The vertex arising from the triangle anomaly leads to higher-order nonrenormalizable divergences, so the model must involve effective interactions that are restrained at some high energy scale. Summing (strong) "bubble" diagrams might restrain contributions to $m_{b}^{2}$ i. e. $\delta m_{b}^{2}\left(p^{2}\right) \sim$ $\left(m_{b}^{2}-p^{2}\right)$ at squared four-momentum $p^{2}$.
} 
because of their interaction with the cosmic microwave background photons. These events could be related to neutral $b^{0}$ arriving from even the largest distances and interacting rather strongly in the atmosphere, if a sufficient flux of $b^{0}$ at $\sim 10^{20} \mathrm{eV}$ is attainable. We estimate below that a flux of $\sim 10^{-20}\left(\mathrm{~cm}^{2}-\mathrm{s}-\mathrm{sr}\right)^{-1}$ is attainable for $b^{0}$ originating in the decay of massive inflatons which we have shown 13 can constitute much of dark-matter, and for which we have calculated [13] a lifetime orders of magnitude greater than the present age of the universe. 2. Over many years, a number of unusual events of a particular kind [6, 7] at high energies have been observed in several photo-emulsion chamber experiments at different high-altitude locations [7]. These events at $E_{\text {lab }} \sim 10^{16}-10^{17} \mathrm{eV}$ involve double-core $\gamma$-families, where a $\gamma$-family consists of a bundle of high-energy particles incident on the chamber in almost the same direction. In the laboratory, the direction is in the very forward region for the collisions. Recently [6, , 8, 8], these events have been analyzed in terms of high transverse momentum jet production in QCD. The result of this analysis [6, 7, 8], which must be viewed in the context of limited statistics, is stated to be an order of magnitude excess of events at the largest inferred [8] transverse momenta. It is said to be suggestive of new physics above $\sim 10^{16} \mathrm{eV}$ characterized by particle production in the forward direction with unusually large transverse momenta. 8] $F^{2}$ In fact, an early summary of cosmic rays [4] already presented a number of events involving "binocular" families, analysis of which indicated an unusual general characteristic of forward production at transverse momenta of some tens of $\mathrm{GeV}$ of a decaying entity with an effective mass of tens of $\mathrm{GeV}$. Subsequent data [5] indicated family events at energies above $10^{16} \mathrm{eV}$ characterized by large production transverse momenta, and a rich hadron content. We show below that the cross section for production of $Z^{\prime}$ by $b^{0}$ must be dynamically enhanced at the largest values of the squared four-momentum transfer to a nuclear target. There is a large deep-inelastic cross section. Decay of $Z^{\prime}$ into quark and antiquark and quark ejection from the target (followed by fragmentation and secondary hadronic interactions) naturally gives rise to events having two cores with large transverse momenta in forward directions (and also possibly to three such cores 漹).

To clearly see the dynamical features, consider production of $Z^{\prime}$ in a $b^{0}$-quark collision via exchange of $Z^{\prime}$, as in the diagram in Fig. 1a. The interaction at the upper vertex is $\left(\frac{g^{2}}{2 \pi^{2} F}\right)\left(\frac{1}{4} \epsilon_{\mu \nu \sigma \rho} F_{Z^{\prime}}^{\mu \nu} F_{Z^{\prime}}^{\sigma \rho}\right)$; at the lower vertex $g\left(\bar{\psi}_{q} \gamma^{\mu} \psi_{q} Z_{\mu}^{\prime}\right)$. The differential cross section is then

$$
\frac{d \sigma}{d(\cos \theta)} \cong\left(\frac{g^{2}}{2 \pi}\right)\left(\frac{g^{2}}{2 \pi^{2} F}\right)^{2} \frac{\frac{s^{2}}{4}\left(\sin ^{2} \theta+\frac{1}{4}(1-\cos \theta)^{3}\right)}{\left(\frac{s}{2}(1-\cos \theta)+m_{Z^{\prime}}^{2}\right)^{2}}
$$

The mass $m_{Z^{\prime}}$ (taken as $\sim m_{Z}$ simply for numerical illustration) is retained only in the propagator (to avoid singular behavior in the squared four-momentum transfer $\left.-t=\frac{s}{2}(1-\cos \theta)\right)$. For c. m. energy $\sqrt{s} \sim 1 \mathrm{TeV}\left(E_{\text {lab }}^{b} \sim 10^{17} \mathrm{eV}\right.$ on $m_{q} \sim 5 \mathrm{MeV}$ ), the total cross section is large, $>10 \mathrm{mb}$. This is the first feature, which is due to the low scale $F$. The second feature is the marked enhancement at the largest $|t|$, which is caused by the numerator function in Eq. (1). Fas Most of $\sigma$ arises from $|t| \geq$ $m_{Z^{\prime}}^{2}$. These two features carry over to the realistic situation involving deep-inelastic interactions of $b^{0}$ impinging upon nucleons in the atmosphere, as in the diagram in Fig. 1b. In terms of the usual parton model variables $x=\frac{|t|}{2 m_{N}\left(E_{b}-E_{Z^{\prime}}\right)}, y=$ $\frac{\left(E_{b}-E_{Z^{\prime}}\right)}{E_{b}}$, and the nucleon structure function $F_{2}(x,|t|)$, the laboratory differential

\footnotetext{
${ }^{2}$ When discussing unusual behavior in the forward direction, it is important to recognize that the cross section for single diffractive dissociation must tend to zero at extremely high energies, as the interaction of hadrons approaches that of black disks. See 14

F3 This behavior is also present in the cross section for the inverse Primakoff effect, which involves production of a photon by an incident pseudoscalar boson in a nuclear Coloumb field. See [15]
} 
cross section is

$$
\frac{d^{2} \sigma_{b}}{d x d y}=\left(\frac{g^{2}}{4 \pi}\right)\left(\frac{g^{2}}{2 \pi^{2} F}\right)^{2} \frac{\frac{s}{8}|t|}{\left(|t|+m_{Z^{\prime}}^{2}\right)^{2}} \frac{F_{2}}{2}\left(1+(1-y)^{2}+\frac{y^{2}}{2}\right)
$$

with $s \cong 2 m_{N} p_{b},|t| \cong 4 p_{b} p_{Z^{\prime}} \sin ^{2} \frac{\theta}{2}$. We have retained $m_{Z^{\prime}}$ only in the propagator, $m_{N}$ is the nucleon mass, $p_{b}, p_{Z^{\prime}}$ are momenta, and $\theta$ is the angle of $Z^{\prime}$. The total cross section is greater than $10^{-2} \mathrm{mb}$. This is a large deep-inelastic cross section, because it is controlled by $\left(\frac{1}{2 \pi^{2} F}\right)^{2}$. Compare Eq. (2) to the usual cross section for deep-inelastic scattering of a charged lepton via photon exchange, whose size is controlled by $\sim \frac{1}{|t|}$

$$
\frac{d^{2} \sigma_{\ell}}{d x d y}=\frac{\left(e^{2}\right)^{2}}{4 \pi} \frac{s}{\left(|t|^{2}\right)^{2}} \frac{F_{2}}{2}\left(1+(1-y)^{2}\right)
$$

In this comparison, one sees again the enhancement at the largest $|t|$ due to the numerator in Eq. (2). The double-core events do indicate a fall off consistent with $\left(\frac{1}{p_{T}^{2}}\right)$, not $\left(\frac{1}{p_{T}^{4}}\right)$, at the largest inferred [8] transverse momenta $p_{T}$. The two features are just what is required to deal with the two unusual empirical aspects of the high-energy cosmic-ray interactions, if sufficient flux of $b^{0}$ here is achievable.

Consider first $b^{0}$ near to the maximum energy, which we consider to be $\sim$ $10^{20} \mathrm{eV}$. The maximum energy of $b^{0}$ arises from their possible origin in the decay of inflatons $\phi, \phi \rightarrow b b$. We have calculated the $\phi$ mass in detail 13 in a specific phenomenological cosmological model. It is in a limited range $\sim 10^{19}-\sim 10^{20} \mathrm{eV}$. The mass range is based upon explicit calculation of inflaton potentials which exhibit $\underline{\text { both }}$ a minimum at an energy scale $\phi_{c} \cong 10^{16}-10^{17} \mathrm{GeV}$ and a maximum at the Planck scale. 13, 16. We have shown that the massive inflaton quanta produced near the end of inflation can constitute much of dark matter today, with an energy density estimated to be in the range $0.1-0.5$ of critical. The inflaton may not be completely stable. In the model [13], it has no coupling to ordinary matter except for a possible very weak coupling to neutrinos proportional to neutrino mass; this leads to the decay $\phi \rightarrow \nu_{\tau} \bar{\nu}_{\tau}$ with a lifetime given by ${ }^{F 4}$

$$
\tau_{\phi}=\left(\Gamma_{\phi}\right)^{-1} \sim\left(\left(10^{-5}\right)\left(\frac{m_{\nu_{\tau}}}{\phi_{c}}\right)^{2} \frac{m_{\phi}}{8 \pi}\right)^{-1} \cong 10^{26} \mathrm{~s}
$$

where we use here $m_{\nu_{\tau}} \cong 0.06 \mathrm{eV}$ as a hypothetical heaviest-neutrino mass, and our calculated [13 values $\phi_{c} \cong 10^{17} \mathrm{GeV}, m_{\phi} \cong 5 \times 10^{10} \mathrm{GeV}$. A possible "mixing" between the chiral-like dynamics ${ }^{F 5}$ which we examined in the cosmological model [13], and a possible low-scale chiral dynamics involved in neutrino mass suggests an effective interaction $f(\phi b b)$, with the order of magnitude of $\mathrm{f}$ given by

$$
f \sim\left(\sqrt{10^{-5}} m_{\nu_{\tau}}\right)\left(\frac{m_{\nu_{\tau}}}{4 \pi^{2} F}\right)
$$

This results in a definite branching ratio, $\left(\Gamma(\phi \rightarrow b b) / \Gamma\left(\phi \rightarrow \nu_{\tau} \bar{\nu}_{\tau}\right)\right)=r \sim 0.5 \times$ $10^{-4}$. We have estimated [18, 19, 13, that the decay of dark matter can give rise to significant flux of the maximum-energy neutrinos here, about $2 \times 10^{-16}\left(\mathrm{~cm}^{2}-\right.$ $\mathrm{s}-\mathrm{sr})^{-1}$ from within our galaxy alone. The estimate for $r$ thus implies a flux

\footnotetext{
F4 The factor $\sqrt{10^{-5}} \sim\left(\frac{1}{30 \pi^{2}}\right)$ arises from a (conservative) estimate of the contribution to the model matrix element from a neutral, massive intermediate state which "mixes" into $\nu_{\tau} \bar{\nu}_{\tau}$ with an effective coupling proportional to $\left(\sqrt{m_{\nu_{\tau}} / \phi_{c}}\right)^{2}$.

$F 5$ The chiral dynamics suggests a reason for the necessary smallness of the inflaton quartic self-coupling, $\lambda \sim 10^{-13}$ which leads to $m_{\phi} \cong \sqrt{\lambda} \phi_{c} \sim 5 \times 10^{10} \mathrm{GeV}$.

${ }^{F 6}$ In a chiral $\sigma$-model, $b^{0}$ couples to neutrinos with strength $\left(m_{\nu} / F\right)$, i. e. note 17
} 
of $b^{0}$ of $\sim 10^{-20}\left(\mathrm{~cm}^{2}-\mathrm{s}-\mathrm{sr}\right)^{-1}$. In fact, the recent AGASA data [i] consists of a handful of "hadron-like" events, all close to $10^{20} \mathrm{eV}$, in an exposure of about $2.6 \times 10^{20}\left(\mathrm{~cm}^{2}-\mathrm{s}-\mathrm{sr}\right)$. We have given numerical examples of a possible "bump" structure 19] in events near to the maximum energy, that is a structure above the GZK cut-off and near to $10^{20} \mathrm{eV}$.

The anomalous double-core events [8] imply a flux of $b^{0}$ of the order of $10^{-16}\left(\mathrm{~cm}^{2}-\right.$ $\mathrm{s}-\mathrm{sr})^{-1}$ at say $\sim 10^{16.5} \mathrm{eV}$. These could be produced from the inverse of the process shown in Fig. 1a. The $Z^{\prime}$ would have to be produced in exceedingly dense matter in order to interact before decay. The unique possibility would seem to be the densities reached by neutron-star matter. This suggests neutron stars as discrete sources for at least some of the unusual high-energy cosmic rays. In this respect, it is worth noting that there is recent evidence [3] for anisotropy in the arrival directions for cosmic rays around $10^{18} \mathrm{eV}$, with a significant excess near the directions of the galactic center and the Cygnus region [3. The excess flux is at the level of $\sim 10^{-18}\left(\mathrm{~cm}^{2}-\mathrm{s}-\mathrm{sr}\right)^{-1}$. Among the events above $10^{19} \mathrm{eV}$, there is a curious tendency to clustering of directions [2]. One significant cluster is said [2] to encompass the direction of a known pulsar, as well as that of the Cygnus "loop". Of course, protons may be accelerated to high energies near magnetized neutron stars.F7F8

The ideas in this paper illustrate the fact that the absence of events characterized by new dynamics at the LHC, for $p p$ collisions at c. m. energies $\sim 10 \mathrm{TeV}$, would not refute the presence of new phenomena in cosmic-ray events at $E_{\text {lab }}>10^{16} \mathrm{eV}$. Evidently, the particular nature of an incident particle can be crucial, as well as the energy which it brings into the collision process. The corollary is that the cosmicray experiments and the coming experiments sensitive to fluxes of very high energy neutrinos (above $10^{19} \mathrm{eV}$ ) are essential [20]. The comment in this last paragraph could apply also to cosmic-ray Centauro (and anti-)events, not detected at present accelerators. Coalescence of the final-state $Z^{\prime}$ and an atmospheric target excited to mass of $\sim m_{Z^{\prime}}$ might be a uniquely favorable dynamical configuration for the transient formation of a coherent state of pions 21. This state could result in Centauro-type characteristics with $\left\langle n_{\pi}\right\rangle \sim 2\left\langle n_{\pi}\right\rangle \gtrsim 2\left\langle n_{\pi}\right\rangle_{Z} \gtrsim 56$, and $\left\langle p_{T}^{2}\right\rangle^{1 / 2} \sim$ $\sqrt{\frac{2}{3}}\left(\frac{m_{Z^{\prime}}}{\left\langle n_{\pi}\right\rangle_{Z^{\prime}}}\right) \sim \sqrt{\frac{2}{3}}\left(\frac{m_{Z}}{\left\langle n_{\pi}\right\rangle_{Z}}\right) \cong 2.6(\mathrm{GeV} / \mathrm{c})$. The empirical numbers for Centauro I at primary energy of $\sim 1650 \mathrm{TeV}$, are $\sim 74$ and $\sim 1.8(\mathrm{GeV} / \mathrm{c}))$, respectivly [22].

S. B. is grateful to Dr. Patrick Heiliger for much help.

\section{References}

[1] M. Takeda et. al., Phys. Rev. Lett. 81, 1163 (1998)

[2] M. Takeda et. al., astro-ph/9902239, Feb. 1999

[3] N. Hayashida, Y. Fujimoto and S. Hasagawa, astro-ph/9807045, July 1998. This paper also discusses anisotropies around $10^{17} \mathrm{eV}$ reported by the Yakutsk and Haverah Park experiments.

[4] C. M. G. Lattes, Y. Fujimoto and S. Hasagawa, Phys. Rep. 65, 151 (1980) J. Ren et al., Phys. Rev. D38, 1404 (1988)

\footnotetext{
$F 7$ If produced from quarks at accelerator energies, $Z^{\prime}$ would give rise to some "extra" hadronic jets, and possibly to some missing energy in neutrinos.

${ }^{F} 8$ The existence of anisotropy restricted to a narrow range of energies [3] is suggestive of production of neutral particles by higher-energy particles at discrete sources. If these particles are concentrated at a definite energy (as in two-body decay products of a massive particle), then the particular produced particle will have its "typical" energies cut-off both at high values and at low values by the low and high-multiplicity tails of multiplicity distributions, respectively.
} 
[5] Chacaltaya and Pamir Collab., L. T. Baradzei et. al., Nucl. Phys. B370, 365 (1992)

[6] Z. Cao, L. K. Ding, Q. Q. Zhu and Y. D. He, Phys. Rev. Lett. 72, 1794 (1994)

[7] Z. Cao, L. K. Ding, Q. Q. Zhu and Y. D. He, Phys. Rev. D56, 7361 (1997)

[8] Y. D. He, Mod. Phys. Lett. A11, 2013 (1996)

[9] S. L. Adler, Phys. Rev. 177, 2426 (1969)

[10] J. S. Bell and R. Jackiw, Nuovo Cimento 60A, 47 (1969)

[11] K. Greisen, Phys. Rev. Lett. 16, 748 (1966)

[12] G. T. Zatsepin and V. A. Kuzmin, Zh. Eksp. Teor. Fiz. 4, 114 (1966) ( JETP Lett. 4, 78 (1966))

[13] S. Barshay and G. Kreyerhoff, Eur. Phys. J. C5, 369 (1998)

[14] M. L. Good and W. D. Walker, Phys. Rev. 120, 1857 (1960)

S. Barshay, P. Heiliger, and D. Rein, Z. Phys. C56, 77 (1992)

[15] S. Barshay, H. Faissner, R. Rodenberg and H. de Witt, Phys. Rev. Lett. 46, $1361(1981)$

[16] S. Barshay and G. Kreyerhoff, Z. Phys. C75, 167 (1997); Erratum Z. Phys. C76, 577 (1997)

[17] S. Barshay, Mod. Phys. Lett. A6, 3583 (1991)

[18] S. Barshay and G. Kreyerhoff, Nuovo Cimento 112A, 1469 (1999). This paper considers some speculations concerning these neutrinos as a cosmological energy source.

[19] S. Barshay and G. Kreyerhoff, Nuovo Cimento 112A, 1463 (1999)

[20] J. Linsley, Proc. $19^{\text {th }}$ Intl. Cosmic-Ray Conf. 3, 438 (1985)

Y. Takahashi, Proc. $24^{\text {th }}$ Intl. Cosmic-Ray Conf. 3, 595 (1995)

[21] S. Barshay and P. Heiliger, Z. Phys. C66 , 259 (1995)

[22] For a recent summeries see: A. Ohsawa and Chacaltaya Emulsion Chamber Collab., in ICRR-Report-434-98-30 and 454-99-12. 


\section{Figures}

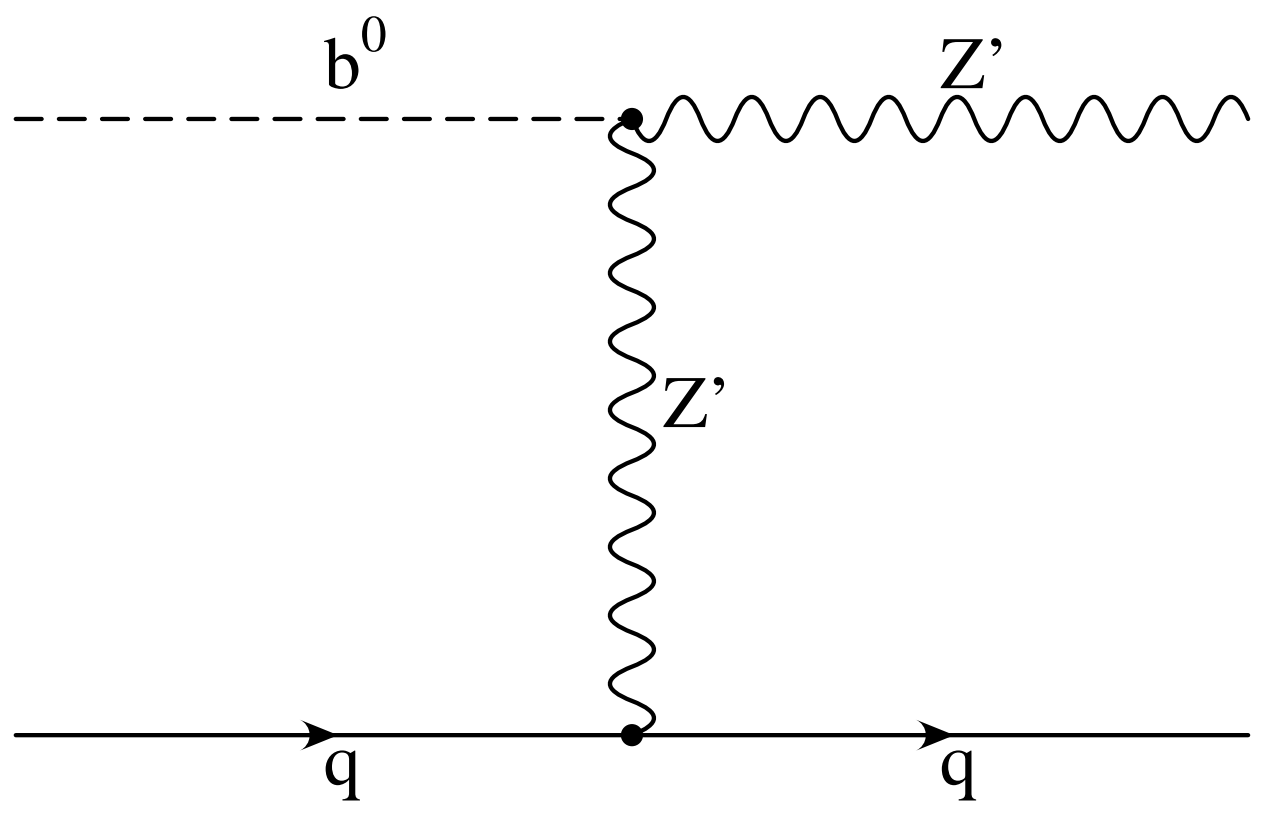

Figure 1a: Production of a neutral massive vector boson $Z^{\prime}$ by a pseudoscalar Goldstone boson $b^{0}$ in collision with a quark $q$.

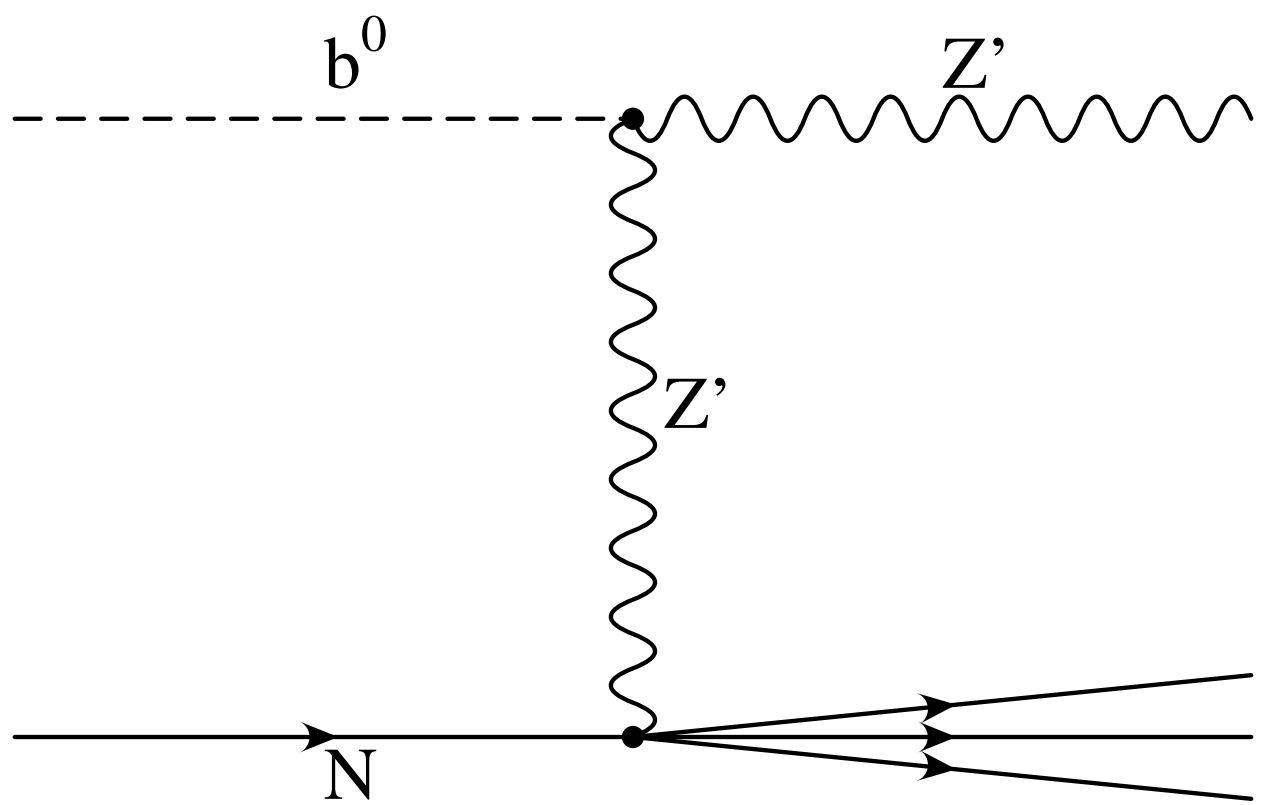

Figure 1b: Deep-inelastic scattering initiated by $b^{0}$ in collision with a nucleon $N$. 